\title{
Umbrales de precipitación basados en intensidad para crecidas torrenciales en la quebrada Manizales, Colombia
}

\author{
$\checkmark$ antonio Castillo Ruales ${ }^{1}$ \\ PHILIPPE CHANG ${ }^{1}$ \\ JORGE JULIÁN VÉLEZ UPEGUI ${ }^{1}$ \\ JEANNETTE ZAMBRANO NÁJERA ${ }^{1}$ \\ FERNANDO MEJÍA FERNANDEZ ${ }^{1}$
}

\section{Resumen}

Los umbrales de precipitación tradicionales, basados en curvas de magnitud-duración, no permiten establecer un tiempo de antelación (TA) adecuado para Sistemas de Alerta Temprana (SAT) sobre cuencas sin información de radar meteorológico y con tiempos de concentración cortos. Este trabajo propone la determinación de umbrales de precipitación independientes de la duración del evento, a partir de la correlación de la precipitación, medida en tiempo real en la parte alta de la cuenca, con los niveles resultantes aguas abajo. El estudio se realizó sobre la quebrada Manizales, Caldas (Colombia), la cual ha presentado eventos de crecidas torrenciales que se han agravado por los procesos y efectos de la urbanización. La modelación hidrológica distribuida en TETIS incluyó patrones locales de distribución de precipitación, y los parámetros hidráulicos del flujo fueron evaluados con el modelo bidimensional IBER, a partir de tres estados de riesgo. Los resultados sugieren que la cuenca es altamente sensible a los cambios de intensidad y no tanto para la magnitud de la lluvia, por lo tanto, se propone una metodología para umbrales en base a la lluvia acumulada móvil de 10 minutos a partir del inicio del evento para ser implementado en SAT de la ciudad de Manizales.

Palabras Clave: Umbrales de precipitación, Crecidas torrenciales, Riesgo de inundación, Sistema de alerta temprana.

\section{Rainfall thresholds based on intensity for flash floods in the Manizales River, Colombia}

\section{Abstract}

Where the traditional approach in establish alert thresholds for Early Warning Systems (EWS) relies on rainfall depth-duration curves, in the context of watersheds characterised by short runoff response time and in the absence of

1 Universidad Nacional de Colombia - sede Manizales

Autor de correspondencia: Castillo Ruales, A. (Antonio): Universidad Nacional de Colombia: Cra 27 \# 64-60 - Bloque J - Lab. Hidráulica, Manizales, Colombia. Correo electrónico: acastillorua@unal.edu.co
Historia del artículo:

Artículo recibido: 28-III-2019/ Aprobado: 15-I-2020

Disponible online: 15 de enero de 2020

Discusión abierta hasta septiembre de 2021 
weather radar data, such practise cannot be implemented effectively. Hence, this research establishes alert threshold based on real-time rainfall measurement correlation over a catchment between headwaters and stages observed downstream in absence of rainfall depth-duration data. The Manizales River catchment (Caldas, Colombia) has presented flash flood events that have been aggravated by urbanization of the area. The distributed hydrological model TETIS was implemented using local rainfall distribution patterns to establish overland flow. The 2DH hydraulic flow model was based on IBER that determined 3 risk levels. The study suggests that then catchment response is highly sensitive to rainfall intensity changes rather than precipitation depth over time. Hence a specific methodology based on 10 minute cumulative mobile rainfall depth over time was used to establish rainfall thresholds for the EWS of the Manizales watershed.

Keywords: Rainfall thresholds, flash floods, Risk flood, Early warning system.

\section{Umbrais de chuva baseados em intensidade para inundações no rio Manizales, Colômbia}

\section{Resumo}

Os umbrais de chuva tradicionais, baseados em curvas de magnitude-duração, não permitem estabelecer um tempo de avanço adequado para Sistemas de Alerta Prévio (SAP) em bacias sem informações de radar meteorológico e com tempos de concentração curtos. Este trabalho propõe a determinação de umbrais de chuva independentes da duração do evento, com base na correlação de precipitação, medida em tempo real a montante da bacia, com os níveis resultantes a jusante. 0 estudo foi conduzido no rio Manizales, Caldas (Colômbia), que apresentou eventos torrenciais de inundação que foram agravados pelos processos e efeitos da urbanização. A modelagem hidrológica distribuída no TETIS incluiu padrões locais de distribuição de precipitação, e os parâmetros hidráulicos do fluxo foram avaliados com o modelo bidimensional IBER, a partir de três estados de risco. Os resultados sugerem que a bacia é altamente sensível a mudanças na intensidade e não tanto pela magnitude da chuva, portanto, propõe-se uma metodologia para umbrais baseada na precipitação acumulada móvel de 10 minutos do início do evento para ser implementado no SAT da cidade de Manizales.

Palavras chave: Umbrais de chuva, Inundações torrenciais, Risco de inundação, Sistema de alerta prévio.

\section{Introducción}

Las crecidas torrenciales son definidas como un tipo de inundación que se caracteriza por una corta duración y un caudal máximo relativamente alto, generalmente como resultado de una alta intensidad de precipitación sobre un área pequeña (NOOA, 2012). Debido a la brevedad en la escala temporal de estos eventos, el pronóstico de las crecidas torrenciales resulta complejo y los tiempos de reacción de las comunidades y organismos se ven limitados, constituyendo una amenaza súbita, destructiva y mortal (Unesco y Omm, 2012). Según la Organización Meteorológica Mundial (OMM), las crecidas torrenciales causan en promedio más de 5.000 muertes al año y causan millones de dólares de pérdidas materiales, convirtiéndolas en la amenaza natural de mayor tasa de mortalidad (OMM, 2008).

La ciudad de Manizales, Caldas (Colombia) no es ajena a los desastres causados por crecidas torrenciales sobre sus quebradas urbanas. Para el periodo comprendido entre los años 2000-2013 se reportaron 3.480 personas afectadas, 415 familias afectadas, 89 viviendas destruidas y 27 muertes a causa de estos desastres hidrometeorológicos (Alcaldía de Manizales, 2014). En especial, el evento de inundación ocurrido el 21 de marzo del 2011 sobre la parte media-baja de la cuenca de la quebrada Manizales dejó 160 personas afectadas, 20 viviendas destruidas y pérdidas materiales significativas sobre la zona industrial de la ciudad, evidenciando el alto riesgo por inundación al que se encuentran expuestas 
las comunidades ribereñas de esta quebrada (Serna, 2012; Quintero, 2013).

En respuesta a estos eventos, las autoridades locales desarrollaron un Plan de Acción Inmediato (PAI) para la cuenca que permite la adaptabilidad, reducción y respuesta frente al riesgo de inundación (Corpocaldas, 2011). El PAI contempló diferentes medidas para la mitigación del riesgo, tanto estructurales: construcción de obras para el control torrencial, como no estructurales: implementación de un Sistema de Alerta Temprana (SAT) por inundación. Para la implementación del SAT, la cuenca de la quebrada Manizales fue instrumentada con una red de estaciones hidrometeorológicas con el propósito de monitorear las condiciones de precipitación en la parte alta de la cuenca y los niveles de la quebrada en la zona urbana (Sánchez, Mejía y Zambrano, 2018). Sin embargo, el SAT de Manizales aún no se encuentra finalizado debido a que no se han definido los umbrales de alerta.

Los umbrales de alerta definen las condiciones hidrometeorológicas bajo las cuales se toma la decisión de emitir las alarmas por parte de los organismos de emergencia hacia las comunidades en riesgo (Sene, 2008). Inicialmente, el concepto de umbral de precipitación fue adoptado por Caine (1980) para determinar la lluvia que desencadena los movimientos en masa sobre las laderas (Montesarchio, et al., 2015). Posteriormente, este concepto se adaptó en los SAT por inundación, definiéndose como: la cantidad de lluvia acumulada para una duración dada, que genera un caudal crítico sobre una determinada sección transversal del río (Georgakakos, 1995). En vista de que las lluvias intensas son la causa más común de las crecidas torrenciales (National Oceanic and Atmospheric Administration NOOA, 2012), los umbrales de alerta están relacionados directamente con la precipitación y existen numerosas metodologías que permiten determinarlos en función de la información disponible, grado de tecnología y complejidad del SAT (Montesarchio, et al., 2015). En general, las metodologías para el cálculo de umbrales de precipitación se basan en la aplicación de métodos empíricos, métodos basados en modelos hidrológicos o métodos probabilísticos (Martina, et al., 2006).

Los métodos empíricos se basan en datos históricos de inundaciones y lluvia que relacionan la ocurrencia del evento con la magnitud y duración de la precipitación crítica por medio de una ecuación potencial (Cannon, et al., 2008; Diakakis, 2012; Montesarchio, et al., 2015). Esta metodología fue usada por (Ávila, Carvajal y Justino, 2015) para la cuenca del Río Cali (Valle del Cauca, Colombia) en donde, a partir de 27 datos históricos de inundaciones y de precipitación diaria, se establecieron umbrales de lluvia acumulada antecedente a largo plazo (5 a 25 días) entre los 73-273 mm. Sin embargo, los umbrales fueron calculados con base en la información disponible de precipitación (diaria y no horaria); por lo tanto, este enfoque se ajusta mejor para ríos con procesos de inundaciones fluviales y no tanto para crecidas torrenciales (Santos y Fragoso, 2016).

La segunda metodología de umbrales parte de la Guía de Crecidas Repentinas (FFG por sus siglas en inglés) propuesta por (Mogil, Monro y Groper, 1978). El método FFG consiste en una modelación hidrológica inversa para determinar la cantidad de lluvia que genere el menor caudal de inundación en la salida de la cuenca. Las alertas se emiten si el umbral es superado por los datos observados en tiempo real o lluvia pronosticada para una duración determinada, siendo necesaria la instrumentación de la cuenca por medio de estaciones meteorológicas o radar (Norbiato, Borga y Dinale, 2009). FFG es ampliamente utilizado y en su evolución ha incorporado la modelación de diferentes formas de hietogramas sintéticos, el uso de modelos espacialmente distribuidos y la condición de humedad antecedente del suelo (Moore, 2002). En los últimos años, los modelos hidráulicos han sido introducidos para que los umbrales sean calculados en función de la geometría del canal y la relación entre el nivel del agua alcanzado y el área de inundación (Wu, et al., 2015; Candela and Aronica, 2016).

La última familia metodológica se basa en métodos probabilísticos que buscan establecer la dependencia entre la lluvia y las posibles consecuencias (en términos económicos) sobre una sección del río (Golian, Saghafian y Maknoon, 2010). Por lo anterior, los umbrales de precipitación son obtenidos al minimizar dos funciones diferentes: una función de utilidad asociada a la teoría Bayesiana, que permite establecer una medida en la percepción de daño, y una función de riesgo basado en el concepto de entropía, que brinda una medida de las incertidumbres dentro del sistema (Martina, Todini y Libralon, 2006). Por lo tanto, la anterior metodología está diseñada para simplificarle a los tomadores de decisiones el proceso de emisión de alarmas, permitiendo 
evaluar el costo/beneficio de activar o no los protocolos de respuesta del SAT.

La aplicación de los métodos empíricos en la cuenca quebrada Manizales está limitada por la falta de una base de datos de eventos históricos de inundación que permita establecer la lluvia detonante de crecidas torrenciales. De igual manera, series temporales hidrometeorológicas de corta duración (3 años) impiden un análisis estadístico exhaustivo para el uso de los métodos probabilísticos. Por lo anterior, el uso de modelos hidrológicos constituye la opción más adecuada para la determinación de los umbrales en la cuenca de estudio.

De acuerdo con (Golian, et al., 2011), para cuencas pequeñas y de respuesta rápida es más importante el cambio de la precipitación en el tiempo como desencadenante de inundaciones que la precipitación total acumulada. Por lo anterior, se propone en este artículo el cálculo de umbrales de precipitación alternativos para cuencas pequeñas y de alta montaña, basados en el cambio de la intensidad e independientes de la duración del evento, obtenidos a partir de un modelo hidrológico distribuido y un modelo hidráulico bidimensional, con el objetivo de ser adoptados en el SAT de la ciudad de Manizales.

\section{Materiales y Métodos}

\section{1. Área De Estudio}

El área de estudio comprende la cuenca de la Quebrada Manizales, localizada al Oriente de la capital del Departamento de Caldas, Colombia entre las coordenadas geográficas $5^{\circ} 02^{\prime}-5^{\circ} 04^{\prime}$ sobre la latitud Norte y $75^{\circ} 23-75^{\circ} 29$ longitud Oeste. La cuenca presenta una superficie de $32 \mathrm{~km}^{2}$, con una distribución aproximada del 56\% de área rural sobre la parte alta de cuenca y un $44 \%$ de área urbana en la parte baja. La parte alta de la cuenca se caracteriza por fuertes pendientes (entre el 23 y $67 \%$ ), la existencia de zonas de reserva forestal y el desarrollo de actividades agropecuarias y mineras. Por otra parte, en la zona baja se encuentra la zona industrial de la ciudad (Aristizábal, 2015) y los asentamientos urbanos como: Maltería, Verdum, Bajo Juanchito y San Marcel, que se encuentran en alto riesgo de inundación (Quintero, 2013).

Figura 1. Localización de la cuenca Quebrada Manizales y red de estaciones hidrometeorológicas

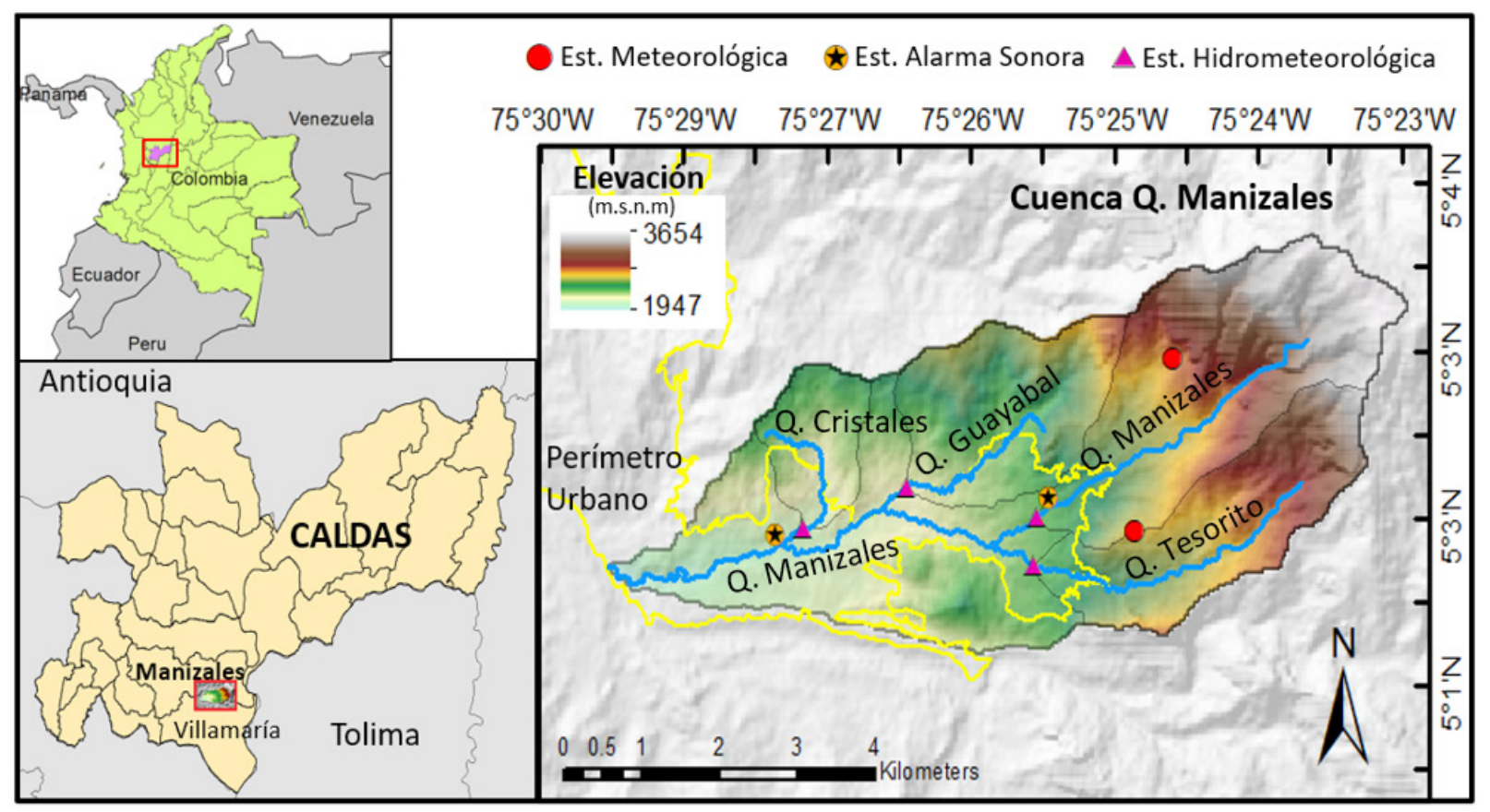


La Quebrada Manizales nace sobre los 3.266 m.s.n.m. y recorre $12,8 \mathrm{~km}$ a través de la cuenca con una pendiente promedio del 10\%, para desembocar finalmente sobre el río Chinchiná a los 1.947 m.s.n.m. (Corpocaldas, 2011). La cuenca se encuentra instrumentada con 4 estaciones hidrometeorológicas (que incluyen pluviometría) en los afluentes: quebrada Tesorito, Cristales y Guayabal; además, se tienen dos estaciones meteorológicas sobre la parte alta de la cuenca y dos estaciones de alarma sonora en puntos estratégicos de los centros poblados (ver Figura 1). Para el análisis hidrológico de este estudio se dividió la cuenca en 4 subcuencas: quebrada Tesorito, Manizales, Guayabal y Cristales (Figura 1).

Los datos de precipitación y niveles a escala cincominutal fueron obtenidos del Centro de Datos e Información Ambiental de Caldas (CDIAC), que se pueden descargar del website cdiac.manizales.unal. edu.co (IDEA y Corpocaldas, 2015) administrados por el Sistema Integrado de Monitoreo Ambiental de
Caldas (SIMAC) (ver Tabla 1). El modelo hidrológico se trabajó con el Modelo de Elevación Digital (MED) de la NASA (resolución 30 m), y la información detallada de la geología, suelos y vegetación de la cuenca se proporcionan en (Corpocaladas, 2012) (ver Figura 2). Para el modelo hidráulico se utilizó el MED de la quebrada en su parte urbana, obtenido a partir del levantamiento LIDAR de la ciudad de Manizales, con una resolución de 1,0 m (AEROESTUDIOS S.A, 2014). Además, a partir de la ortofotografía de la ciudad se construyó el mapa de usos del suelo, definiéndose 7 clases asociadas a un coeficiente de rugosidad de Manning.

\subsection{Metodología para definición de} umbrales de precipitación

La metodología se divide en tres componentes principales que son: la conceptualización de la cuenca, la determinación del comportamiento hidráulico de la quebrada ante diferentes escenarios y la determinación de los umbrales.

Figura 2. A) Modelo de elevación digital cuenca quebrada Manizales con resolución 30 m. B) Mapa de pendientes cuenca en grados. C) LIDAR quebrada Manizales parte urbana con resolución 1,0 m. D) Franja uso de suelos para la asignación de los coeficientes de rugosidad de Manning

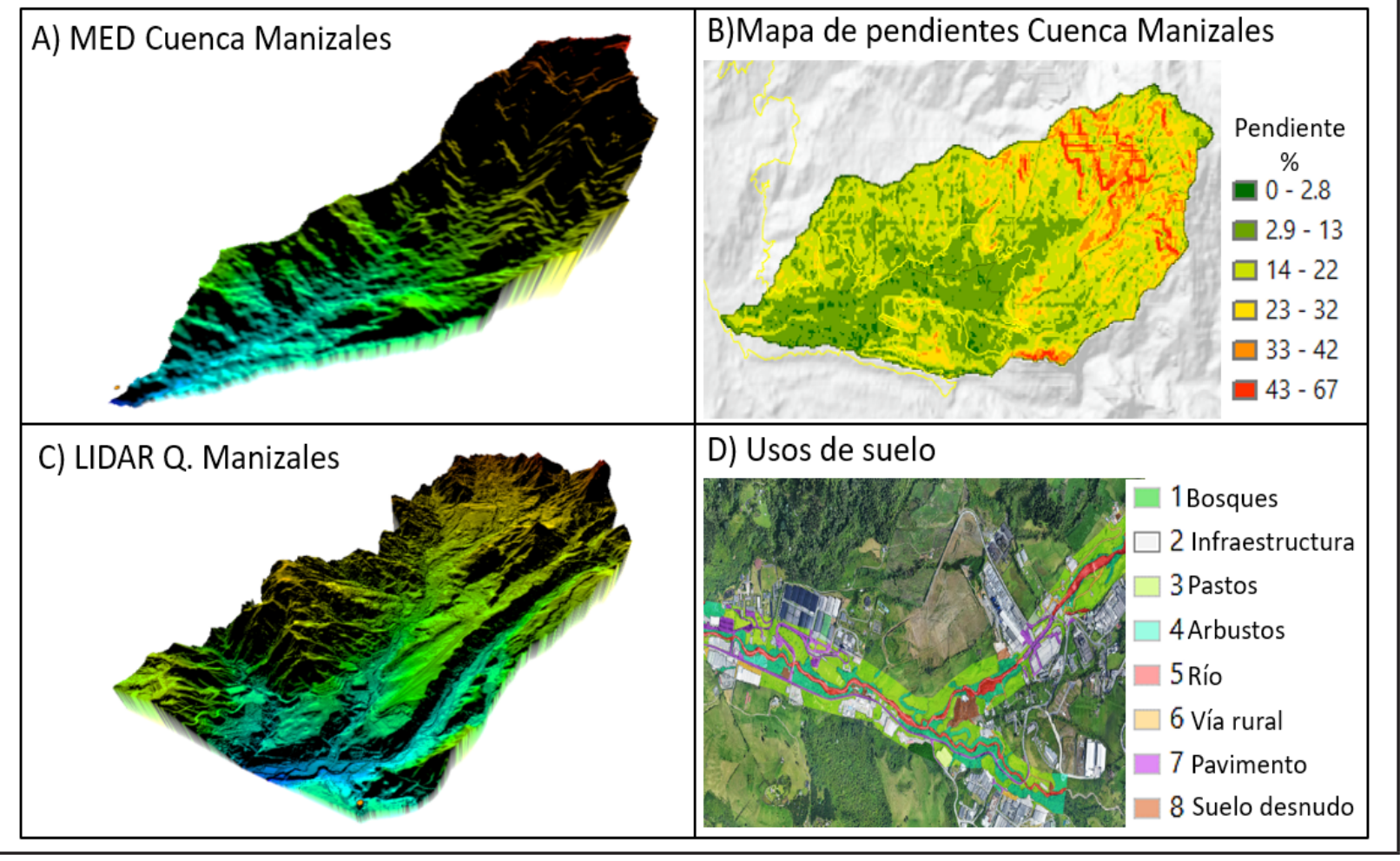

ISSN 1794-1237 / Volumen 17 /Número 33 / Enero-Junio 2020 / Reia33003 pág 1-16 
TABLA 1. LISTADO DE ESTACIONES UBICADAS EN LA QUEBRADA MANIZALES UTILIZADAS PARA LA DEFINICIÓN DE UMBRALES

\begin{tabular}{l|c|c|c|c|c}
\multicolumn{1}{c|}{ Estación } & & Tipo & Año de instalación & Latitud & Longitud \\
\cline { 5 - 6 } & & & N & W \\
\hline Finca La Paz & Plu-1 & M & 2014 & $5^{\circ} 2^{\prime} 3.93^{\prime \prime}$ & $75^{\circ} 25^{\prime} 9.27^{\prime \prime}$ \\
\hline Hacienda Manzanares & Plu-2 & M & 2014 & $5^{\circ} 3^{\prime} 16.2^{\prime \prime}$ & $75^{\circ} 24^{\prime} 40.68^{\prime \prime}$ \\
\hline Q. Manizales-Skinco & Niv-2 & H & 2014 & $5^{\circ} 2^{\prime} 19.18^{\prime \prime}$ & $75^{\circ} 25^{\prime} 49.08^{\prime \prime}$ \\
\hline Q. Guayabalito & Niv-1 & H & 2010 & $5^{\circ} 2^{\prime} 15.9^{\prime \prime}$ & $75^{\circ} 26^{\prime} 3.26^{\prime \prime}$ \\
\hline Q. Cristales-Valles del Alhambra & Niv-4 & H & 2015 & $5^{\circ} 2^{\prime} 29.15^{\prime \prime}$ & $75^{\circ} 26^{\prime} 44.45^{\prime \prime}$ \\
\hline
\end{tabular}

Nota: M=Estación meteorológica - H= Estación hidrometeorológica

Figura 3. Conceptualización de la cuenca de la quebrada Manizales con la distribución de las estaciones de monitoreo para el SAT

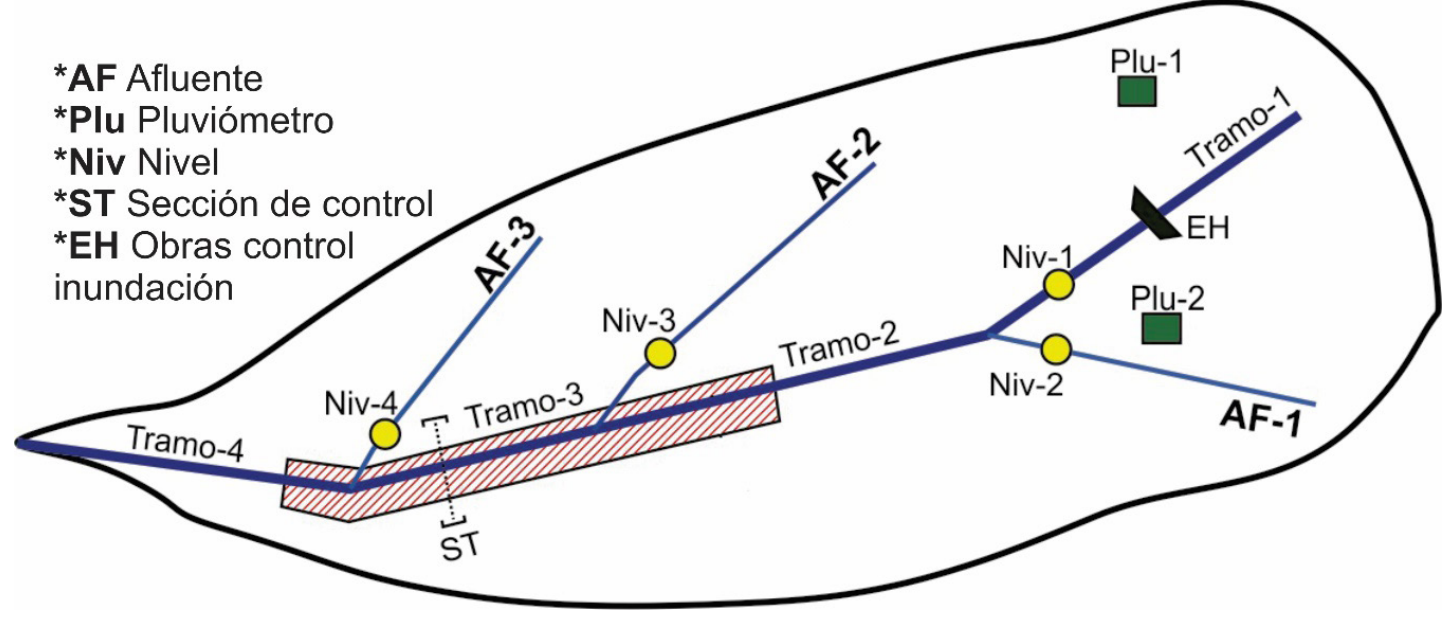

El primer proceso de conceptualización de la cuenca consistió en determinar las zonas críticas o de monitoreo. Para tal fin, la cuenca se esquematizó como se muestra en la Figura 3. La quebrada fue dividida en cuatro tramos: 1) el Tramo-1 ubicado sobre la parte alta de la cuenca, en donde existe la presencia de obras de control torrencial (diques transversales, EH en la figura), finaliza en el punto de afluencia de la quebrada Tesorito (Tramo Afluente 1 o AF-1). La parte alta de la quebrada Manizales y la quebrada Tesorito constituyen el mayor aporte de caudal y se encuentran instrumentadas por las estaciones hidrometeorológicas Niv-1 y Niv-2 respectivamente. 2) El Tramo-2 va desde la confluencia del tramo 1 y el afluente 1 hasta la entrada del afluente 2 o quebrada Guayabal. Este tramo se caracteriza por la presencia de zonas de riesgo de inundación en el sector cercano a la descarga del afluente AF-2 (quebrada Guayabal). 3) El Tramo-3 va desde donde termina el tramo 2 hasta la entrada de la quebrada Cristales o afluente 3. Este tramo es el más crítico por la gran cantidad de comunidades y zonas industriales asentadas en la rivera de la quebrada y, finalmente, 4) el Tramo-4 que inicia en el punto de descarga del afluente AF-3 (quebrada Cristales) y culmina en la salida de la cuenca. Este tramo presenta zonas de riesgo en la parte inicial del tramo.

Aunque los afluentes AF-2 y AF-3 están debidamente instrumentados por las estaciones hidrometeorológicas Niv-3 y Niv-4, el aporte de caudal de estas quebradas es muy bajo porcentualmente, por lo que no son críticos para la generación de las crecidas 
torrenciales. Las estaciones meteorológicas de la parte alta de la cuenca (Plu-1 y Plu-2) fueron determinadas como las estaciones de disparo a partir de la información de precipitación. De manera que se concluyó que la zona marcada en rojo en la Figura 3 será la zona de alerta específicamente para la sección de control transversal ST crítica, y las estaciones que detonarán la alerta serán Plu-1 y Plu-2.

La segunda etapa de la metodología consistió en determinar el comportamiento hidráulico de la quebrada y la tercera etapa consistió en correlacionar la precipitación con los niveles obtenidos en la modelación hidráulica. Para este fin, se siguió la metodología propuesta por (Montesarchio, et al., 2009) con las siguientes modificaciones: la introducción de un modelo hidráulico bidimensional para evaluar diferentes niveles de agua sobre la sección crítica y la determinación de umbrales de precipitación independientes de la duración del evento, es decir, en función de la intensidad instantánea de la precipitación; en la Figura 4 se muestra el esquema metodológico propuesto en este trabajo y a continuación se explica cada una de las etapas.
El proceso se inició con la selección de los datos y parámetros de entrada, es decir, las lluvias a considerar y las condiciones de saturación del suelo. Las lluvias fueron establecidas para los periodos de retorno (Tr) de 2,33, 15, 40 y 100 años. La distribución espacial de la precipitación para cada periodo de retorno se obtuvo de la modelación hidrológica en TETIS realizada sobre la cuenca del río Chinchiná (Corpocaladas, 2012). La duración del evento fue definida igual al tiempo de concentración, el que a su vez fue calculado por (Vélez \& Botero, 2011). Se seleccionaron 3 patrones de distribución temporal de la precipitación de acuerdo con la duración del evento, que fueron obtenidos para la ciudad de Manizales por (Rincón, Vélez, \& Chang, 2015). Los anteriores patrones de distribución temporal de precipitación se seleccionaron con el fin de observar el impacto de la distribución del hietograma en el tiempo de respuesta, es decir, observar cómo la ocurrencia del pico de intensidad de la lluvia afecta al hidrograma. Se consideraron las distribuciones más críticas al inicio (distribución I), a la mitad (distribución II) y al final (distribución III) de la duración del evento (Mancini et al., 2002).

Figura 4. Esquema metodológico para la determinación de los umbrales de precipitación

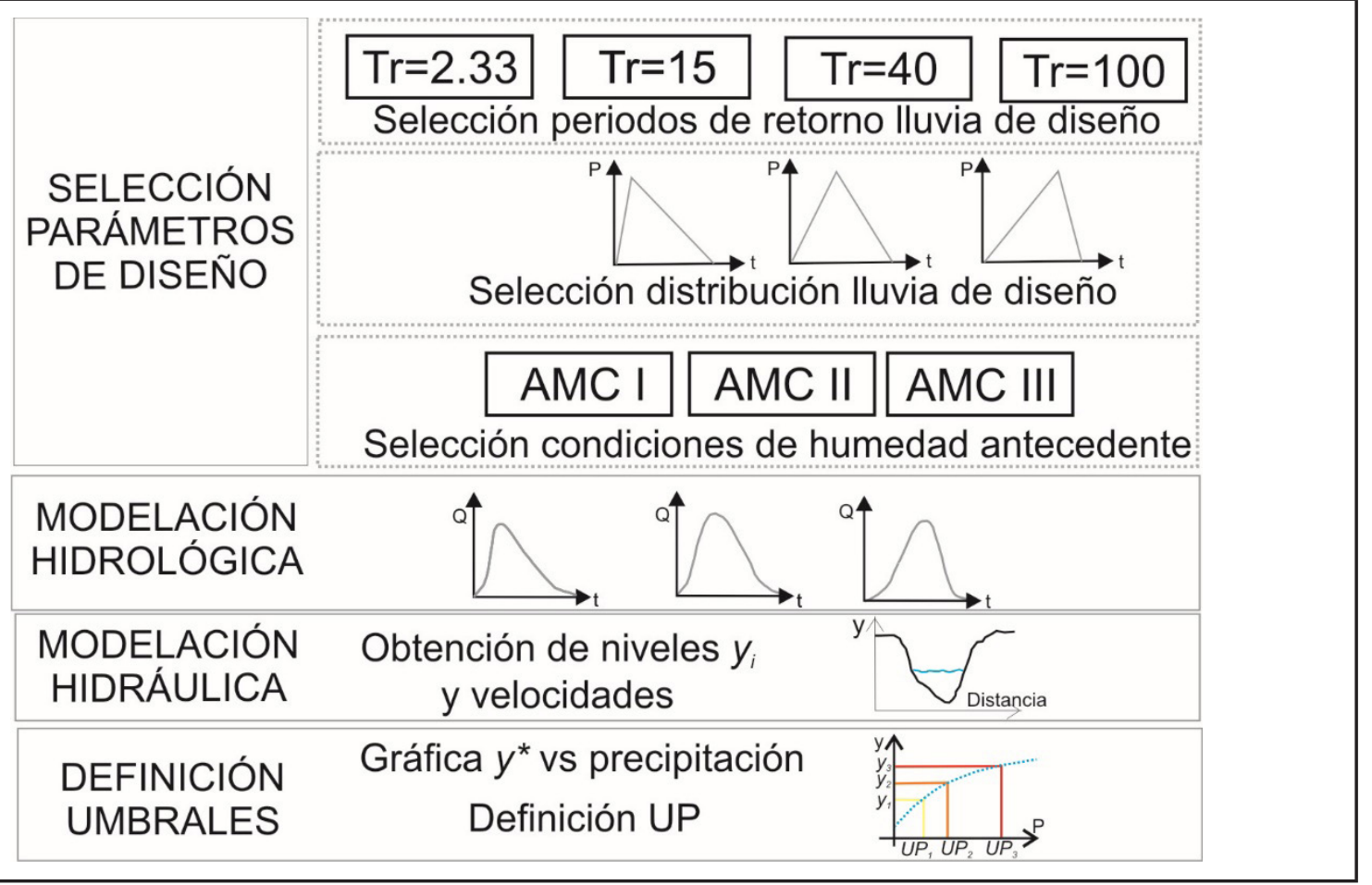


La modelación hidrológica se realizó por medio del modelo distribuido TETIS v8.30b, que es un modelo continuo y/o de evento, conceptual y distribuido, desarrollado por el Departamento de Ingeniería Hidráulica y Medio Ambiente de la Universitat Politécnica de Valencia (DIHMA-UPV). El modelo utiliza la metodología de la onda cinemática acoplada a las características geomorfológicas de la cuenca (Geomorphologic Kinematic Wave-GKW) para la propagación de la onda a lo largo de la red de canales (Vélez, et al, 2009). La información inicial y los parámetros calibrados que el modelo TETIS requiere fueron obtenidos a partir de la modelación realizada en la cuenca del río Chinchiná por (Corpocaladas, 2012), teniendo en cuenta que la cuenca de la quebrada Manizales está contenida en la cuenca del río Chinchiná y, por lo tanto, sólo se realizó una actualización de los datos de entrada para precipitación. Se obtuvieron los hidrogramas de salida en los puntos de estaciones hidrometeorológicas (Niv-1, Niv-2, Niv-3 y Niv-4) para los periodos de retorno seleccionados, a través de la simulación combinada de cada uno de los 3 patrones de distribución de la precipitación y de los 3 escenarios de condición de humedad antecedente (AMC). La Tabla 2 muestra la relación entre los diferentes escenarios de humedad antecedente (Soil Conservation Service, 1986) y los parámetros de estado inicial de TETIS.

TABLA 2. PARÁMETROS ESTADO INICIAL TETIS PARA CONDICIÓN DE HUMEDAD ANTECEDENTE

\begin{tabular}{l|c|c|c|}
\hline Parámetro & AMC I & AMC II & AMC III \\
\hline $\begin{array}{l}\text { H1-Almacenamiento } \\
\text { estático }\end{array}$ & 50 & 80 & 100 \\
\hline $\begin{array}{l}\text { H2-Agua en superficie } \\
\text { H3-Almacenamiento } \\
\text { gravitacional }\end{array}$ & 0 & 0 & 0 \\
\hline $\begin{array}{l}\text { H4-Estado inicial del } \\
\text { acuífero }\end{array}$ & 50 & 50 & 50 \\
\hline $\begin{array}{l}\text { H5-Caudal en el cauce } \\
\text { H6-Intercepción por la } \\
\text { vegetación }\end{array}$ & 20 & 50 & 100 \\
\hline
\end{tabular}

Luego, se realizó la modelación hidráulica mediante el modelo numérico bidimensional de volúmenes finitos IBER, desarrollado por el Grupo de Ingeniería del Agua y del Medio Ambiente, GEAMA (Universidade da Coruña), el Grupo de Ingeniería Matemática (Universidade de Santiago da Coruña), el Instituto Flumen (Univer- sitat Politécnica de Catalunya y el Centre Internacional de Métodes Numérics en Enginyeria) (Cea y Bladé, 2008; Bladé, et al., 2014). Por medio de este, se determinaron los calados y velocidades, así como los niveles en el cauce mediante la simulación de flujo de los hidrogramas calculados anteriormente. Los coeficientes de rugosidad $n$ de Manning se obtuvieron a partir del mapa de usos del suelo que se muestra en la Figura 2. Se obtuvieron 7 clases de usos del suelo para la cuenca y se adoptaron valores iniciales del coeficiente de rugosidad según lo sugerido por (US Army Corps of Engineers, 2010); posteriormente, estos se modificaron en el proceso de calibración. La generación de la malla de cálculo se realizó a través de la herramienta "RTIN" con una red de triángulos de lado mínimo 1,00 m y máximo 15,0 m; adicionalmente, se introdujeron las dimensiones de las obras hidráulicas (6 puentes) presentes en la quebrada.

La calibración del modelo hidráulico se realizó con el evento de precipitación del 07 de noviembre de 2017 (Figura 5), ya que éste generó un incremento considerable en los niveles de la quebrada Manizales (La Patria, 2017). El parámetro de calibración de mayor sensibilidad fue el coeficiente de rugosidad de Manning. El ajuste de los resultados de la modelación se evaluó mediante el criterio de Nash-Sutcliffe sobre los puntos Niv-1 y Niv-2; adicionalmente, se realizó comparación visual de los niveles observados contra los encontrados en el modelo en los Tramos 2 y 3 mediante la recolección de evidencia fotográfica y fílmica. Una vez calibrado el modelo hidráulico, se realizaron las simulaciones de los diferentes escenarios ( 3 hietogramas por cada uno de los 4 periodos de retorno y para cada una de las 3 condiciones de humedad antecedente).

La tercera y última etapa de la metodología, consistente en determinar los umbrales, se realizó a partir de los resultados de las simulaciones de la siguiente manera: se definieron cinco (5) secciones transversales (ST) a lo largo de la zona de riesgo (ver ST en la Figura 3), teniendo en cuenta la afectación y el riesgo generado sobre viviendas, infraestructura y vías cercanas al cauce (Candela y Aronica, 2016). Para cada una de las secciones transversales se definieron 3 estados de alerta denominados $y^{*}$, correspondientes a las siguientes profundidades hidráulicas: a) $y_{1}{ }^{*}=$ $80 \%$ del nivel de banca llena, b) $y_{2}{ }^{*}=$ nivel de banca llena y c) $y_{3}{ }^{*}=$ superado el nivel de banca de llena, con lo que hay afectación a la infraestructura (ver Figura 6). 
Figura 5. Evento de Calibración del 07 de noviembre de 2017. A) Reporte estación Niv-2 (quebrada Tesorito). B) Reporte estación Niv-1 (quebrada Manizales-Skinco)
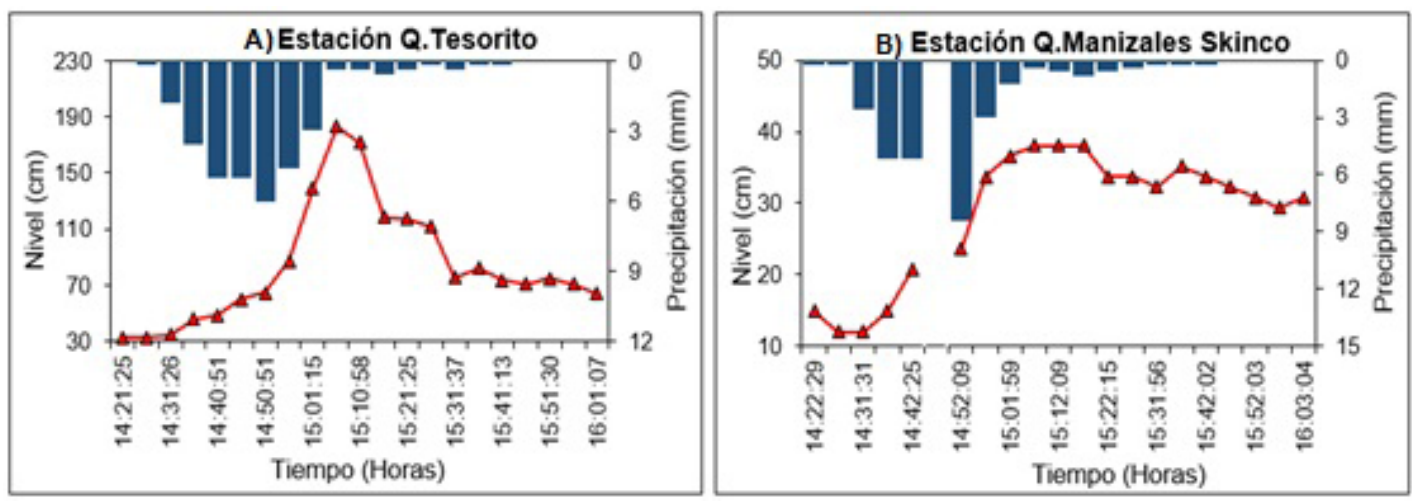

Figura 6. A) Estados de alerta para la sección crítica. B) Esquema para la determinación de los umbrales de precipitación en función de los estados de alerta
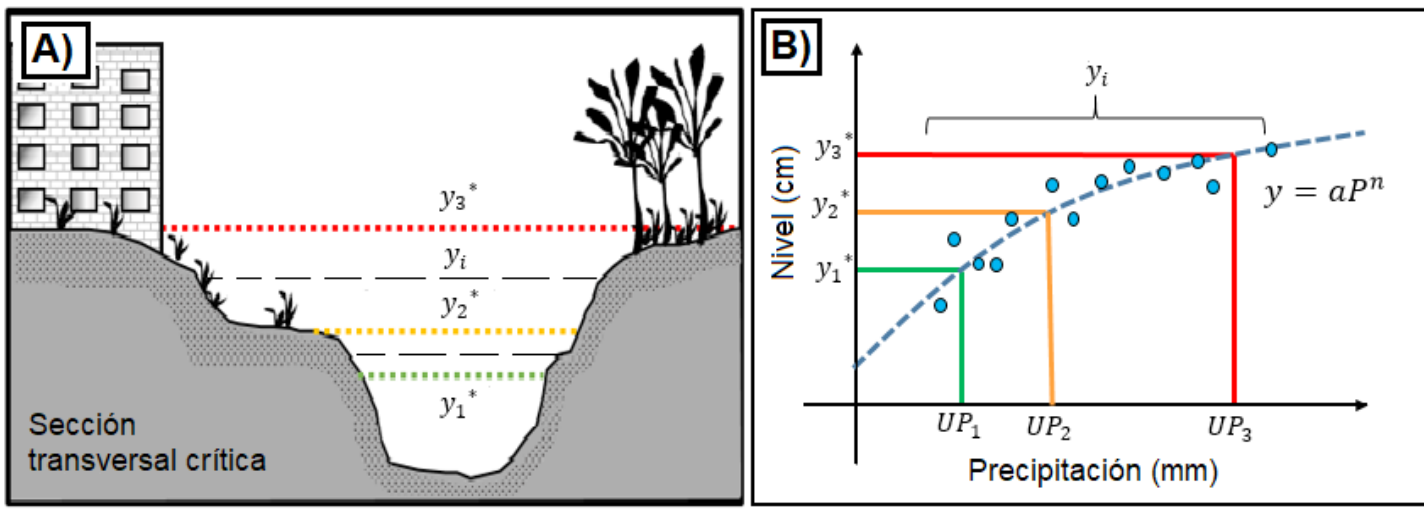

Posteriormente, se seleccionó la sección transversal crítica de la zona de riesgo, definida como la sección en la que primero se genera inundación. Sobre las ST definidas se compararon los niveles simulados para los diferentes escenarios $y_{i}$, es decir, la profundidad hidráulica máxima del escenario $i$ determinado por el patrón del hietograma y las condiciones de humedad antecedente (AMC). Luego, se obtuvo una gráfica con la nube de puntos del conjunto de profundidades $y_{i}$ en función de la precipitación máxima cincominutal de cada hietograma simulado para el respectivo escenario $i$. A partir de la gráfica, se obtuvo una línea de tendencia potencial para el cálculo de los umbrales de precipitación mediante la interpolación gráfica. Los valores de los umbrales corresponden a los puntos de intersección entre los estados de alerta $y_{1}^{*}, y_{2}{ }^{*}$ y $y_{3}{ }^{*}$ sobre la curva y luego su proyección en el eje de las abscisas o precipitación (ver Figura 6).
Por lo tanto, se definieron tres umbrales de precipitación $U P_{1}, U P_{2}$ y $U P_{3}$ vinculados a los tres estados de alerta $y^{*}$ de la sección crítica.

En esta etapa, adicionalmente, se tuvo en cuenta el tiempo de antelación para emitir la alerta (TA), que es definido como la diferencia entre el instante que se alerta a la comunidad y el instante de ocurrencia del evento de inundación. En un SAT por inundación un TA alto es lo deseable de manera que la comunidad tenga tiempo suficiente para resguardarse, y este factor constituye una medida de la precisión y habilidad de predicción en el desempeño del sistema. El TA para los SAT de crecidas torrenciales se ve considerablemente limitado debido a la formación del caudal pico en un corto tiempo después de iniciado el evento de precipitación, menores a $3 \mathrm{~h}$ para cuencas entre los 5-10 $\mathrm{km}^{2}$ (Collier, 2007). 
Teniendo en cuenta lo anterior, en las cuencas que presentan tiempos de crecida $\left(t_{c}\right)$ menores a la duración $(d)$ de la precipitación, los umbrales tradicionales basados en curvas Intensidad-Duración (curvas ID) no permiten obtener un TA que dé tiempo a evacuar. Lo anterior se resume en un escenario donde $t_{c}<d<$ TA (Figura 7A), y por lo tanto no se tendría un TA que permita la evacuación de las personas.

En consecuencia, este trabajo propone el cálculo de umbrales independientes a la duración $(d)$ del evento para un escenario que cumpla la condición $T A<t_{c}<d$. Para cumplir la condición mencionada, se supuso como el TA mínimo igual al tiempo de retardo $\left(t_{\text {lag }}\right)$, definido como el tiempo desde el centro de gravedad del hietograma hasta el pico del hidrograma (Figura 7B). Por esta razón, el indicador propuesto es la precipitación acumulada en ventanas móviles de 10 minutos $\left(P_{10}\right)$. El indicador $P_{10}$ se calcula desde el inicio de la lluviay se compara con los diferentes umbrales $U P_{i}$ para el mismo instante de tiempo, según los datos delas estaciones Plu-1, Plu-2; si $P_{10}>U P_{i}$ se emitirán las alarmas a la población en riesgo para su respectiva evacuación.

Finalmente, la variable $P_{10}$ y los umbrales $U P_{i}$ propuestos fueron evaluados mediante el cálculo del número de disparos $\left(\sum P_{10}>U P_{i}\right)$ para las series temporales de precipitación del año 2017. Aunque esta frecuencia de disparo no es un indicador para evaluar la confiabilidad del SAT en conjunto, permite determinar factores de desempeño como: falsas alarmas, alarmas omitidas, racha de éxitos, tiempo de antelación del sistema y tiempo de respuesta de la comunidad frente a eventos de inundación; además, sí permitió dar una idea de la magnitud de los umbrales fijados y los eventos de precipitación que se presentan en la ciudad. Por lo tanto, si se presentara un número de disparos alto sobre el periodo de análisis, los umbrales serían propensos a dar falsas alarmas, mientras que si se tiene un número de disparos nulo los umbrales serán propensos a omitir alarmas.

\section{Resultados y Discusión}

Los resultados de la modelación hidrológica (Figura 8) muestran que los mayores aportes al caudal total $Q_{T}$ de salida de la cuenca están dados por la quebrada Manizales en el Tramo-1 (en promedio el $38,3 \%$ del $Q_{T}$ en la estación Niv-1) y el AF-1 (en promedio $31,7 \%$ del $Q_{T}$ en la estación Niv-2), mientras que el 30\% del $Q_{T}$ restante se debe al aporte de los afluentes AF-2 $\left(20,6 \%\right.$ del $Q_{T}$ en Niv-3) y AF-3 $\left(9,4 \%\right.$ del $Q_{T}$ en Niv- 4$)$. Esto implica que los caudales generados en la cabecera de la cuenca son los de mayor preponderancia en el desencadenamiento de las inundaciones sobre la zona de riesgo. Sin embargo, las obras de control torrencial (EH), que permiten el amortiguamiento de la onda de crecida sobre el Tramo-1, atenúan los impactos en los Tramos 2-3-4 aguas abajo; por lo tanto, es la quebrada AF-1 la que toma mayor relevancia en el incremento del riesgo por inundación sobre los tramos en mención. En este sentido, las estaciones Plu-1, Plu-2 que se encuentran sobre la parte alta de la cuenca son las de mayor importancia en la activación de las alarmas; además, como respaldo de cada una se utilizarán las estaciones de nivel Niv-1 y Niv-2, que también miden precipitación.

Figura 7. Escenarios TA para el caso de estudio de acuerdo: A) Métodos tradicionales basados en curvas ID. B) Metodología propuesta

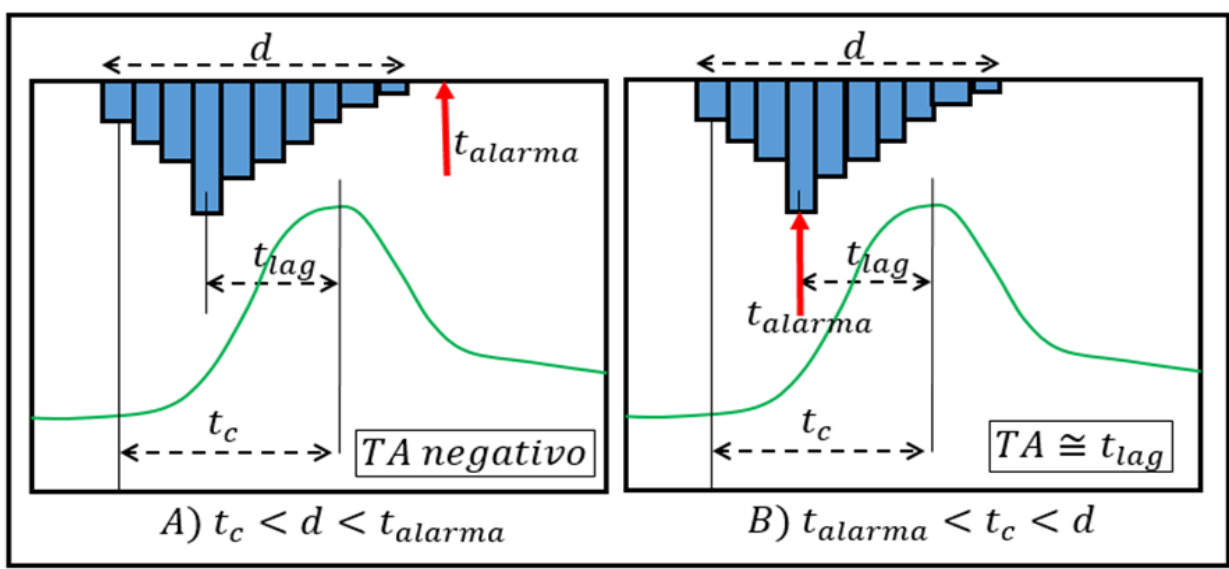


Figura 8. Hidrogramas de salida en cada estación para los diferentes periodos de retorno y patrón de distribución de la precipitación, bajo la condición de humedad antecedente AMC III

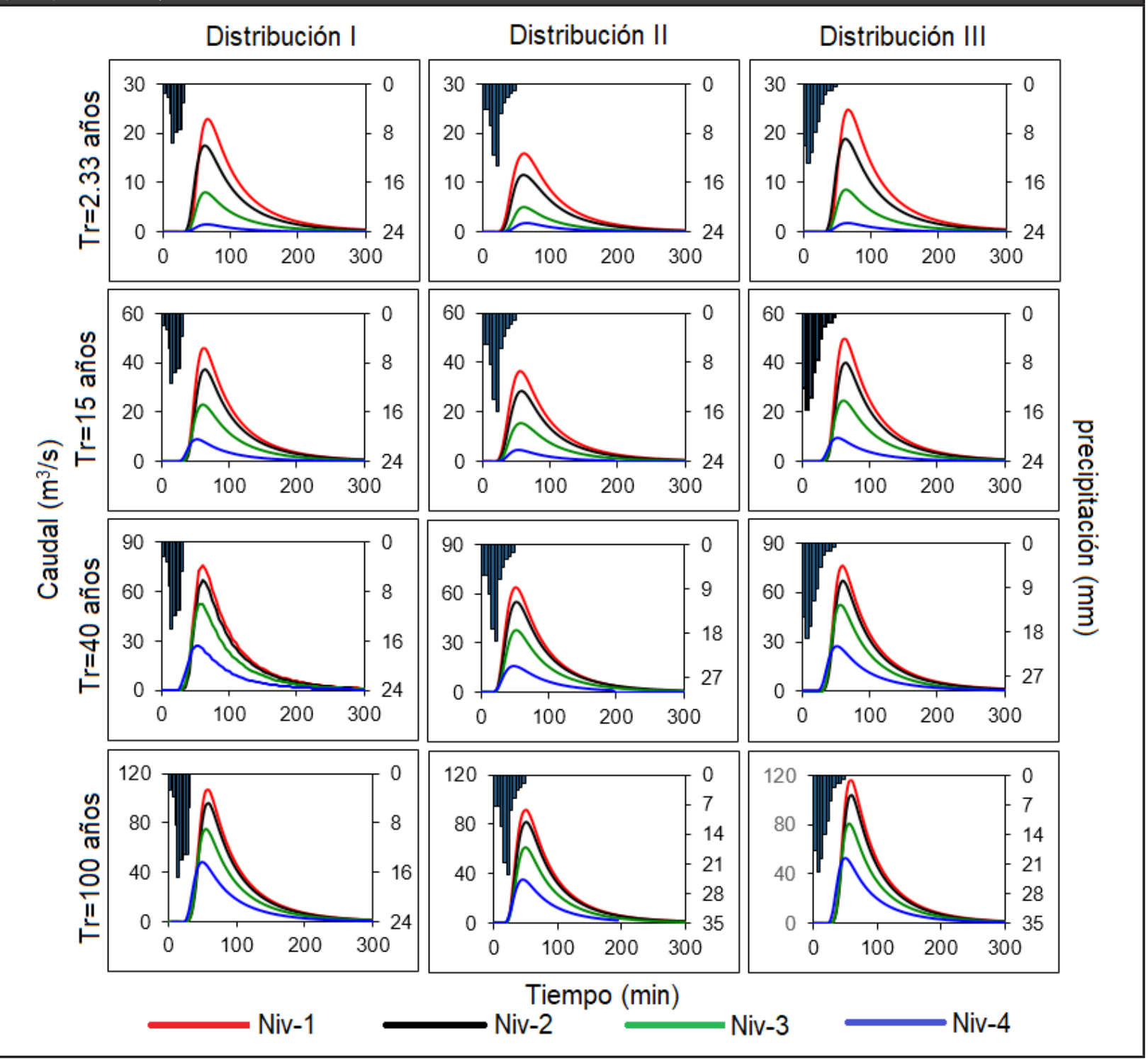

La simulación correspondiente a la calibración de modelo hidráulico muestra que la estación ubicada en la Q. Tesorito (Niv-2) tiende a subestimar los niveles, debido a que no representa adecuadamente los niveles iniciales de la quebrada, ya que se obtuvo un nivel máximo simulado de $144 \mathrm{~cm}$ frente a un nivel observado de $183 \mathrm{~cm}$, obteniéndose un error porcentual de 21\% y una eficiencia de Nash-Sutcliffe de E=0,68. El tiempo al pico se simula correctamente, aun cuando el máximo de los niveles observados es más acentuado, generando una curva de recesión que el modelo sobrestima. Para la parte media de la cuenca, se realizó una calibración visual de los resultados obtenidos por el modelo y de la evidencia fílmica del evento; se analizaron dos secciones transversales en donde se muestra que el modelo refleja adecuadamente las condiciones del flujo, aunque presenta también una subestimación en los niveles.

En cuanto al tiempo desde inicio de la precipitación hasta el pico del hidrograma o $t_{c}$, se obtienen los mayores valores para la distribución I (53,8-62,5 
min) y la distribución III (55,0-61,3 min), aunque no presentan diferencias significativas con respecto a la distribución II (52,5-56,8 min). Lo anterior significa que el caudal pico se presenta casi siempre con la duración del evento de precipitación, o inclusive para algunos casos se presenta que $t_{c}<d$ como se muestra en el evento del 07/11/2017. Por lo anterior, es importante resaltar que el indicador $P_{10}$, al ser independiente a la duración del evento, es un evaluador adecuado para el disparo de los umbrales de alerta ya que garantiza un TA mínimo de reacción en cuencas pequeñas y de montaña que no cuentan con información de radar ni modelos de predicción de lluvia.

En la Tabla 3 se muestran el tiempo de desfase entre los tiempos pico del hietograma y el hidrograma, $t_{\text {lag }}$ y el tiempo en alcanzar el pico en el hidrograma, $t_{c^{\prime}}$ para cada una de las estaciones y distribuciones de precipitación, promediado bajo los diferentes periodos de retorno. El $t_{\text {lag }}$ constituye el TA del SAT, y varía entre los 33-53 min. El patrón de distribución II de la precipitación muestra el escenario más desfavorable en la emisión de las alarmas, puesto que los valores obtenidos del $t_{\text {lag }}$ son menores (32,5-36,3 min) en comparación con el patrón de distribución I (33,0-42,5 min) y la distribución III (45-52,5 min). Esto significa un menor TA para la respuesta de las comunidades, debido a un centro de gravedad del hietograma (ubicado en la mitad de la duración del evento para este caso) más cercano al caudal pico del hidrograma; es decir, el TA se ve reducido cuando las intensidades más altas de precipitación se presentan sobre la mitad de la duración del evento.

Por otra parte, las estaciones muestran que los caudales máximos se presentan en la distribución III (varían entre los $30,7 \mathrm{~m}^{3} / \mathrm{s}$ para un $\mathrm{Tr}=2,33$ años y los $115,6 \mathrm{~m}^{3} / \mathrm{s}$ para un $\mathrm{Tr}=100$ años), siendo aproximadamente un 8\% mayores a los resultados obtenidos con la distribución I y un 33\% con respecto a la distribución II. Esto significa que los hietogramas con intensidades altas de precipitación sobre el final o inicio del evento presentan condiciones más críticas en cuanto al riesgo de inundación. Por lo tanto, se puede concluir que la distribución II es el escenario crítico en relación con el $\mathrm{TA}$, puesto que presenta un menor tiempo de respuesta para la activación de las alarmas, aunque con caudales menores a las demás distribuciones, mientras que la distribución III es el escenario más crítico en la gene- ración de caudales pico y representan un mayor riesgo sobre la población.

Adicionalmente, los niveles simulados con el modelo hidráulico muestran que se producen inundaciones para todos los periodos de retorno considerados. Las zonas más críticas se presentan en la parte media de la cuenca (Tramo-3) donde se encuentran la zona industrial de la ciudad y la vía Panamericana asentadas sobre la llanura de inundación, generalmente en puntos con estructuras hidráulicas que causan un estrechamiento del canal, el represamiento y desborde del mismo (ver Figura 9). Las inundaciones sobre esta zona se presentan básicamente debido al aporte de caudales del AF-1 (quebrada Tesorito) y el Tramo-1 (la parte alta de la quebrada Manizales). Los niveles máximos obtenidos para el canal fueron entre 2,92 $\mathrm{m}$ para el $\mathrm{Tr}=2,33$ años y 5,54 m para el $\mathrm{Tr}=100$ años sobre la zona de riesgo analizada (final Tramo-2, Tramo-3 e inicio Tramo-4); sin embargo, los niveles máximos se alcanzaron en las obras de control torrencial (EH) sobre la quebrada Manizales con un valor de 9,42 m para un $\mathrm{Tr}=100$ años. Además, debido a las pendientes pronunciadas de la quebrada, se obtuvieron velocidades del flujo elevadas $(5,25 \mathrm{~m} / \mathrm{s}-9,37 \mathrm{~m} / \mathrm{s})$ implicando un peligro adicional para las comunidades.

Por último, los umbrales propuestos para la quebrada son: el primer nivel de alerta $U P_{1} 11,2 \mathrm{~mm}$, el segundo nivel de alerta $U P_{2}, 12,8 \mathrm{~mm}$ y el tercer nivel de alerta $U P_{3}, 15,2 \mathrm{~mm}$. En la Figura 10 se evalúa el desempeño de los umbrales propuestos para el evento del 07 de noviembre de 2017, obteniéndose un resultado favorable al presentar el disparo del primer umbral a las 14:45:52, mientras que el nivel pico se registró a las 15:07:12, generando un TA para la activación de las alarmas de 22 minutos.

Adicionalmente, se estimó el número de disparos de dichos umbrales (ver Tabla 4) para la serie de precipitación del año 2017, obteniéndose que: en la estación Plu-1 o Finca La Paz el primer umbral fue superado 1 vez para el evento del 19/03/2017, mientras que el evento del 07/11/2017 produjo que el segundo umbral de alerta se disparara dos veces, y este mismo evento también produjo un disparo en el tercer umbral de alerta. En cuanto a las estaciones Plu-2, Hacienda Manzanares y Niv-1, Quebrada Tesorito se observa un comportamiento similar en el número de disparos para el periodo de aná- 
lisis. Sin embargo, para la estación Niv-3 Q. Manizales Skinco el número de disparos del tercer umbral crece considerablemente (10 disparos). A partir del análisis de la anterior situación se encontró que el número de disparos se produjo para 5 eventos diferentes; sin embargo, se evidenció que este umbral fue superado por valores atípicos de la precipitación cincominutal en 4 de los 5 eventos, teniéndose reportes de más de 34 $\mathrm{mm}$ en 5 minutos, lo que puede suponer una falla en la transmisión por parte de los instrumentos de medición que genera un reporte incorrecto de los datos y no del indicador propuesto.
Figura 10. Validación de los umbrales propuestos para el evento del 07 de noviembre de 2017

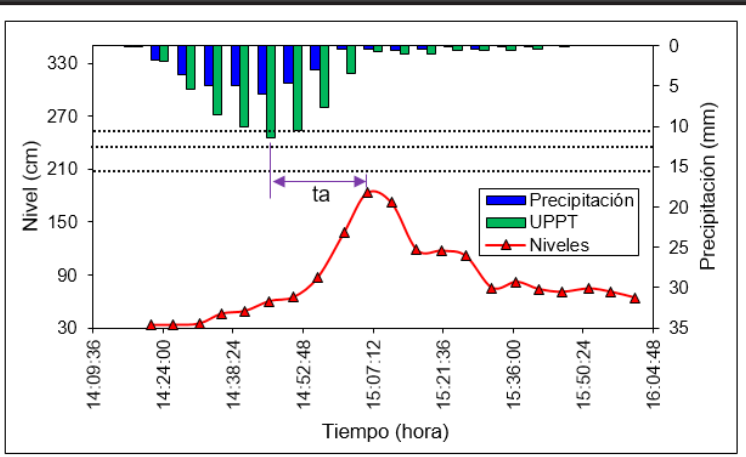

TABLA 3. CÁLCULO TIEMPO DE CRECIDA Y TIEMPO DE RETARDO DE LOS HIDROGRAMAS PARA LAS DIFERENTES ESTACIONES

\begin{tabular}{c|c|c|c|c|c|c|c|}
\hline & & \multicolumn{2}{|c|}{ Distribución I } & \multicolumn{2}{c|}{ Distribución II } & \multicolumn{2}{c|}{ Distribución III } \\
\hline \multirow{2}{*}{ Estación } & & $\mathbf{t}_{\text {lag }}(\min )$ & $\mathbf{t}_{\mathbf{c}}(\mathrm{min})$ & $\mathbf{t}_{\text {lag }}(\mathrm{min})$ & $\mathbf{t}_{\mathbf{c}}(\mathrm{min})$ & $\mathbf{t}_{\text {lag }}(\min )$ & $\mathbf{t}_{\mathbf{c}}(\mathrm{min})$ \\
\hline \multirow{2}{*}{ Niv-1 } & promedio & 42,5 & 62,5 & 33,8 & 53,8 & 51,3 & 61,3 \\
\cline { 2 - 9 } & Desviación estándar & 2,5 & 2,5 & 4,1 & 4,1 & 4,1 & 4,1 \\
\hline \multirow{2}{*}{ Niv-2 } & promedio & 42,5 & 62,5 & 36,3 & 56,3 & 52,5 & 62,5 \\
\cline { 2 - 9 } & Desviación estándar & 2,5 & 2,5 & 4,1 & 4,1 & 2,5 & 2,5 \\
\hline \multirow{2}{*}{ Niv-3 } & promedio & 38,8 & 58,8 & 35,0 & 55,0 & 48,8 & 58,8 \\
\cline { 2 - 8 } & Desviación estándar & 4,1 & 4,1 & 3,5 & 3,5 & 4,1 & 4,1 \\
\hline \multirow{2}{*}{ Niv-4 } & promedio & 33,8 & 53,8 & 32,5 & 52,5 & 45,0 & 55,0 \\
\cline { 2 - 8 } & Desviación estándar & 4,1 & 4,1 & 5,6 & 5,6 & 6,1 & 6,1 \\
\hline
\end{tabular}

Figura 9. Mapas de márgenes de inundación para los diferentes Tr sobre Tramo-3 de la cuenca

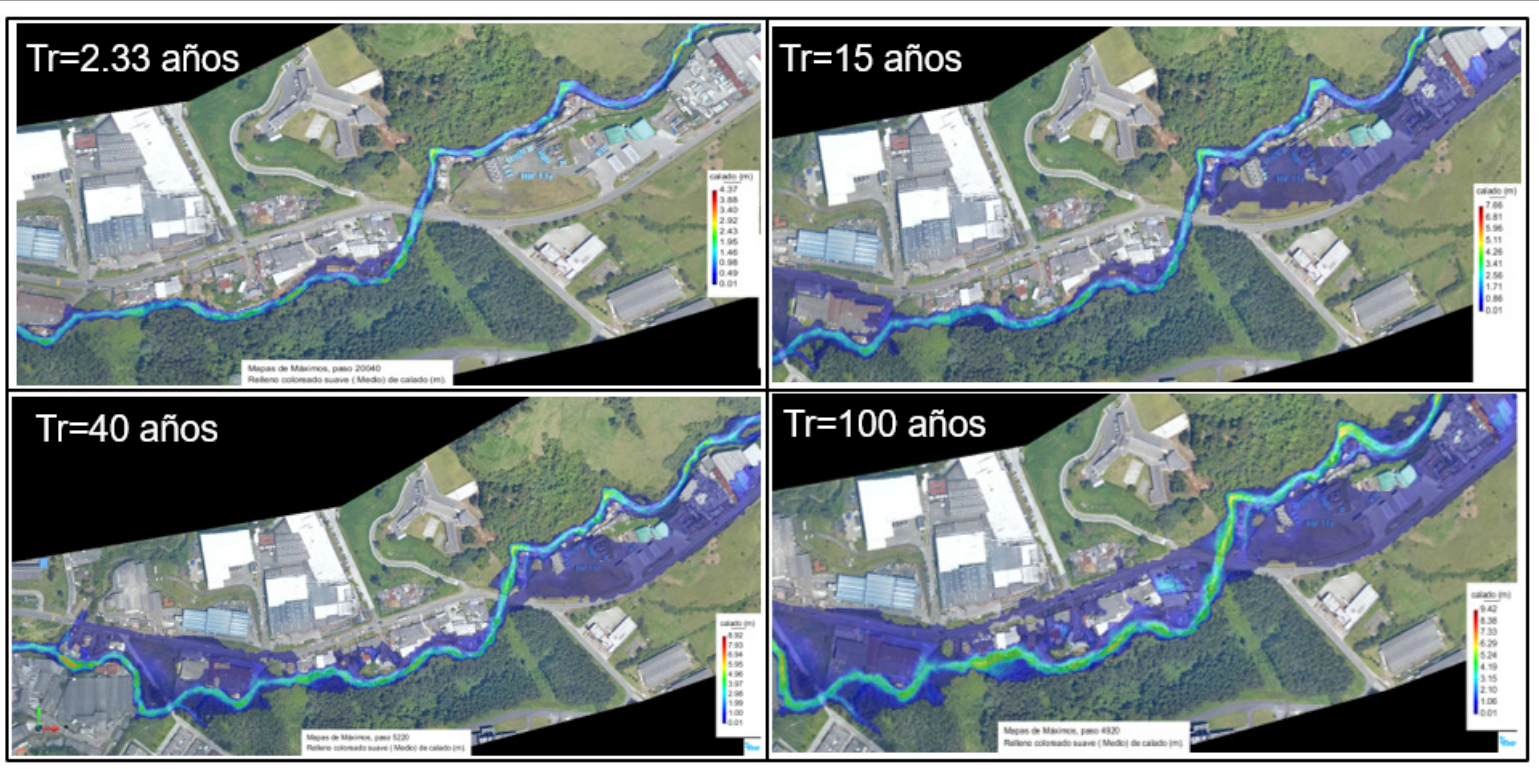


TABLA 4. RESUMEN NÚMERO DE DISPAROS DE UMBRALES PARA LA CUENCA QUEBRADA MANIZALES

\begin{tabular}{l|c|c|c|c} 
& & \multicolumn{3}{|c}{ Número de disparos } \\
\hline Estación & & $\begin{array}{c}\text { Umbral } \\
\mathbf{1}\end{array}$ & $\begin{array}{c}\text { Umbral } \\
\mathbf{2}\end{array}$ & $\begin{array}{c}\text { Umbral } \\
\mathbf{3}\end{array}$ \\
\hline Finca La Paz & Plu-1 & 1 & 2 & 1 \\
\hline $\begin{array}{l}\text { Hacienda } \\
\text { Manzanares }\end{array}$ & Plu-2 & 2 & 2 & 2 \\
\hline $\begin{array}{l}\text { Q. Tesorito } \\
\text { Q. Manizales } \\
\text { Skinco }\end{array}$ & Niv-2 & 3 & 0 & 0 \\
\hline
\end{tabular}

\section{Conclusiones}

Los umbrales de precipitación que desencadenan las crecidas torrenciales se pueden calcular mediante métodos empíricos, con base en modelos hidrológicos o métodos probabilísticos. Este trabajo se enfocó en la determinación de la precipitación crítica evaluada por medio de un modelo hidrológico distribuido combinado con un modelo hidráulico bidimensional para establecer los umbrales con base en unos niveles de riesgo definidos; sin embargo, no tuvo en cuenta el análisis detallado de las incertidumbres relacionadas con la precipitación que permitan asociar unos valores de confianza a los umbrales calculados (Wu et al., 2015).

Generalmente, los umbrales de precipitación están definidos por una cantidad de lluvia acumulada para una duración de evento dado. Sin embargo, el evento del 07 de noviembre de 2017 muestra que la respuesta de la cuenca es muy rápida y por lo tanto el caudal pico se presenta antes de que el evento de precipitación finalice; como consecuencia, el tiempo de antelación para emitir las alarmas es demasiado corto y la metodología tradicional de umbrales no se adapta bien para cuencas de respuesta rápida, a menos que se utilicen modelos de predicción que requieren información adquirida por un radar meteorológico. (Golian et al., 2011) evidenciaron esta situación para crecidas torrenciales sobre cuencas pequeñas de respuesta rápida, y plantearon que el cambio de la precipitación en el tiempo es más importante que el total de lluvia acumulada como desencadenante de inundaciones. Por lo tanto, los umbrales de precipitación de este trabajo fueron calculados como la lluvia acumulada móvil en una ventana temporal de 10 minu- tos. La metodología propuesta permite identificar los cambios en la intensidad de precipitación que detonan las crecidas torrenciales con un tiempo de antelación de 20 minutos para cuencas pequeñas sin modelos de pronósticos de lluvia. Si bien el tiempo de antelación es reducido para implementar acciones que permitan reducir el riesgo por parte de las comunidades, es suficiente para generar la evacuación de los habitantes.

Finalmente, se espera automatizar estos umbrales preliminares de precipitación a las plataformas digitales del CDIAC con el fin de generar alarmas automáticas. Además, se estableció un periodo de prueba en el SAT de Manizales para evaluar el desempeño de acuerdo con otras metodologías, como la propuesta por Manson (1982).

\section{Agradecimientos}

Este trabajo fue apoyado en la información recopilada durante el desarrollo del convenio interadministrativo 221 del 2016 entre el Instituto de Estudios Ambientales de la Universidad Nacional de Colombia Sede Manizales y la Corporación Autónoma Regional de Caldas (Corpocaldas) para la operación y mantenimiento de las redes hidrometeorológicas del Sistema Integrado de Monitoreo Ambiental de Caldas (SIMAC).

\section{Referencias}

AEROESTUDIOS S.A (2014) Informe de campo. Apoyo Geodésico. Manizales.

Alcaldía de Manizales (2014) Gestión del Riesgo Memoria Histórica 2001 - 2013, Gestión del Riesgo Manizales. Available at: http://www.gestiondelriesgomanizales. com/index.php?option=com_content\&view $=$ articl e\&id=102\%3A2001-2013\&catid=45\%3A memoria historica\&Itemid=221 (Accessed: 26 November 2018).

Aristizábal, M. (2015) 'La quebrada Manizales, quebrada ambientalmente por la legalidad e ilegalidad de los vertimientos', Summa luris, 3(1), pp. 207-233.

Ávila, A. D., Carvajal, Y. E. and Justino, F. (2015) 'Representative rainfall thresholds for flash floods in the Cali river watershed, Colombia', Natural Hazards and Earth System Sciences Discussions, 3(6), pp. 4095-4119. doi: 10.5194/nhessd-3-4095-2015.

Bladé, E., Cea, L., Corestein, G., Escolano, E., Puertas, J., Vázquez-Cendón, E., Dolz, J. and Coll, A. (2014) 'Iber: herramienta de simulación numérica del flujo en ríos', 
Revista Internacional de Métodos Numéricos para Cálculo y Diseño en Ingeniería, 30(1), p. 10.

Candela, A. and Aronica, G. T. (2016) 'Rainfall thresholds derivation for warning pluvial flooding risk in urbanised areas', E3S Web of Conferences, 7, p. 18016. doi: 10.1051/e3sconf/20160718016.

Cannon, S. H., Gartner, J. E., Wilson, R. C., Bowers, J. C. and Laber, J. L. (2008) 'Storm rainfall conditions for floods and debris flows from recently burned areas in southwestern Colorado and southern California', Geomorphology, 96(3-4), pp. 250-269. doi: 10.1016/j. geomorph.2007.03.019.

Cea, L. and Bladé, E. (2008) 'Modelización matemática en lecho fijo del flujo en ríos. Modelos 1D y 2D en régimen permanente y variable', in Jornadas Técnicas sobre Hidráulica Fluvial. Madrid: CEDEX-Ministerio de Fomento, pp. 1-30.

Collier, C. G. (2007) 'Flash flood forecasting: What are the limits of predictability?', Quarterly Journal of the Royal Meteorological Society, 133(October), pp. 937-948. doi: 10.1002/qj.

Corpocaladas and IDEA-UNAL (2012) Aplicación de la modelación hidrológica distribuida de tipo conceptual en el departamento de Caldas para eventos extremos. Manizales.

Corpocaladas and IDEA - UNAL (2012) Aplicación de la modelación hidrológica distribuida de tipo conceptual en el departamento de Caldas para eventos extremos. Manizales.

Corpocaldas (2011) Plan de Acción Inmediato Cuenca Quebrada Manizales. Manizales.

Diakakis, M. (2012) 'Rainfall thresholds for flood triggering. The case of Marathonas in Greece', Natural Hazards, 60(3), pp. 789-800. doi: 10.1007/s11069011-9904-7.

Georgakakos, K. P. (1995) 'Real-time prediction for flood warning and management', U.S- Italy Research workshop on the Hydrometeorology, Impacts, and Management of Extreme Floods. Perugia (Hydrometeorology, Impact, and Management of Extreme Floods), p. 9.

Golian, S., Saghafian, B., Elmi, M. and Maknoon, R. (2011) 'Probabilistic rainfall thresholds for flood forecasting: evaluating different methodologies for modelling rainfall spatial correlation (or dependence)', Hydrological Processes. Wiley-Blackwell, 25(13), pp. 2046-2055. doi: 10.1002/hyp.7956.

Golian, S., Saghafian, B. and Maknoon, R. (2010) 'Derivation of Probabilistic Thresholds of Spatially Distributed Rainfall for Flood Forecasting', Water Resources Man- agement. Springer Netherlands, 24(13), pp. 3547-3559. doi: 10.1007/s11269-010-9619-7.

IDEA and Corpocaldas (2015) Centro de Datos e Indicadores Ambientales de Caldas (CDIAC), Generador de indicadores de clima.

Mancini, M., Mazzetti, P., Nativi, S., Rabuffetti, D., Ravazzani, G., Amadio, P. and Rosso, R. (2002) 'Definizione di soglie Pluviometrihe di piena per la realizzazione de un sistema di allertamento in tempo reale per il bacino dell'arno a monte di firenze', in XVIII convegno nazionale di idraulica e costruzioni idrauliche, Potenza. Potenza.

Martina, M., Todini, E. and Libralon, A. (2006) 'A Bayesian decision approach to rainfall thresholds based flood warning', Hydrol. Earth Syst. Sci, 10, pp. 413-426.

Mogil, H. M., Monro, J. C. and Groper, H. S. (1978) 'NWS' s Flash Flood warning and Disaster preparedness Programs', Bulletin American Meteorological Society, 59(6).

Montesarchio, V., Lombardo, F. and Napolitano, F. (2009) Rainfall thresholds and flood warning: an operative case study, Hazards Earth Syst. Sci.

Montesarchio, V., Napolitano, F., Rianna, M., Ridolfi, E., Russo, F. and Sebastianelli, S. (2015) 'Comparison of methodologies for flood rainfall thresholds estimation', Natural Hazards, 75(1), pp. 909-934. doi: 10.1007/s11069-014-1357-3.

Moore, R. (2002) 'Aspects of uncertainty, reliability and risk in flood forecasting systems incorporating weather radar', in Bogardi, J. and Kundzewicz, Z. (eds) Risk, reliability and uncertainty and robustness of water resources system. Cambridge: Cambridge University Press, pp. 30-40.

National Oceanic and Atmospheric Administration NOOA (2012) Guía de referencia para sistemas de alerta temprana de crecidas repentinas 2012. Edited by University Corporation for Atmospheric Research. Estados Unidos.

Norbiato, D., Borga, M. and Dinale, R. (2009) 'Flash flood warning in ungauged basins by use of the flash flood guidance and model-based runoff thresholds', Meteorological Applications. Wiley-Blackwell, 16(1), pp. 65-75. doi: 10.1002/met.126.

La Patria (2017) Lluvias causan emergencias en el suroriente de Manizales, La Patria.com.

Quintero, J. J. (2013) Diagnóstico de la gestión integral del riesgo por inundaciones y avenidas torrenciales en ríos urbanos del departamento de Caldas, Universidad Católica de Manizales. Universidad Católica de Manizales. doi: 10.1017/CB09781107415324.004. 
Rincón, D. F., Vélez, J. J. and Chang, P. (2015) 'Spatio-temporal description of the rainfall in the andean city of Manizales (Colombia) for storm design', in 36th IAHR World Congress 28 June - 3 July, 2015, The Hague, the Netherlands. Hague: IAHR, p. 7.

Sánchez, J., Mejía Fernández, F. and Zambrano, J. (2018) 'Sistema Integrado de Monitoreo Ambiental de Caldas - SIMAC', Boletín Ambiental Instituto de Estudios Ambientales IDEA - Sede Manizales 147, May, p. 19.

Santos, M. and Fragoso, M. (2016) 'Precipitation thresholds for triggering floods in the Corgo basin, Portugal', Water (Switzerland), 8(9). doi: 10.3390/w8090376.

Sene, K. (2008) Flood Warnning, Forecasting and Emergency Response. Springer.

Serna, A. (2012) 'A orillas del riesgo en la quebrada Manizales', La Patria, 25 April.

Unesco and Omm (2012) International Glossary of hydrology, Chair, Publications Board. Geneva, Switzerland.

US Army Corps of Engineers (2010) HEC-RAS River Analysis System Hydraulic Reference Manual. Davis.

Vélez, J. J. and Botero, A. (2011) 'Estimación del tiempo de concentración y tiempo de rezago en la cuenca experimental urbana de la quebrada San Luis, Manizales', Dyna. Universidad Nacional de Colombia, 78(165), pp. 58-71.

Vélez, J. J., Puricelli, M., López Unzu, F. and Francés, F. (2009) 'Parameter extrapolation to ungauged basins with a hydrological distributed model in a regional framework', Hydrology and Earth System Sciences, 13, pp. 229-246.

Wu, S. J., Hsu, C. T., Lien, H. C. and Chang, C. H. (2015) 'Modeling the effect of uncertainties in rainfall characteristics on flash flood warning based on rainfall thresholds', Natural Hazards, 75(2), pp. 1677-1711. doi: 10.1007/s11069-014-1390-2.
PARA CITAR ESTE ARTÍCULO / TO REFERENCE THIS ARTICLE / PARA CITAR ESTE ARTIGO /

Castillo Ruales, A.; Chang, P.; Vélez Upegui, J.J.; Zambrano Nájera, J.; Mejía Fernandez, F. (2020). Umbrales de precipitación basados en intensidad para crecidas torrenciales en la quebrada Manizales, Colombia. Revista EIA, 17(33) enero-junio, Reia33003 pág. 1-16. Disponible en: https://doi.org/10.24050/reia.v17i33.1302 